\title{
LA IMPRESIÓN CISTOSCÓPICA FRENTE AL DIAGNÓSTICO HISTOLÓGICO EN LOS TUMORES VESICALES. ¿COINCIDEN?
}

\author{
E. DE LEÓN MORALES, O. ARANGO TORO, J.A. LORENTE GARÍN, \\ R. CORTADELLAS ANGEL, O. BIELSA GALI, A. GELABERT MAS
}

Servicio y Cátedra de Urología. Hospital del Mar. Universidad Autónoma de Barcelona. Barcelona.

Actas Urol Esp. 27 (1): 18-21, 2003

\section{RESUMEN}

“LA IMPRESIÓN CISTOSCÓPICA FRENTE AL DIAGNÓSTICO HISTOLÓGICO EN LOS TUMORES VESICALES. ¿COINCIDEN?”

OBJETIVO: Como control de calidad en un hospital docente como el nuestro y a raíz de publicaciones recientes al respecto, nos propusimos evaluar la correlación existente entre la impresión cistoscópica de los tumores vesicales y el diagnóstico histológico posterior a la resección transuretral (RTU).

MATERIAL Y MÉTODOS: Para darle mayor objetividad al estudio, solicitamos a todos los miembros del servicio (tanto staff como residentes) que, basados en su experiencia describieran, las características endoscópicas de los siguientes grupos de tumores vesicales: superficiales de bajo grado (T.S.B.G.) GI-II Ta-T1, superficiales de alto grado (T.S.A.G.) GIII Ta, y tumores de alto grado y/o invasivos GIII T1-<T2. En un total de 172 pacientes fue valorada la impresión cistoscópica inicial del tumor vesical y se comparó con el diagnóstico histológico tras la RTU.

RESULTADOS: En 172 tumores, la cistoscopia clasificó a 69 casos como T.S.B.G. GI-II Ta-T1, a 40 como T.S.A.G., y a 55 como tumores de alto grado y/o invasivos GIII T1 y T2. Al comparar el diagnóstico histológico, la cistoscopia coincidió en 46 de 69 (66,6\%) (PNS) pacientes como T.S.B.G., en 13 de 40 (32,5\%) (P<0,05) como T.S.A.G., y en 45 de 51 (88,2\%) (PNS) como tumores GIII T1 y/o invasivos. En 15 de 172 la descripción endoscópica no fue concluyente, y en 12 la anatomía patológica fue normal.

CONCLUSIONES: En base a nuestros resultados, consideramos que en un hospital docente es imprescindible el diagnóstico histológico antes de tomar una decisión terapéutica definitiva, ya que la impresión cistoscópica inicial tiene una baja correlación con el diagnóstico histológico. Estamos en desacuerdo con publicaciones recientes que proponen la fulguración endoscópica basándose solamente en la imagen cistoscópica inicial.

PALABRAS CLAVE: Tumores vesicales. Impresión cistoscópica. Diagnóstico histológico.

\section{ABSTRACT}

“THE CYSTOSCOPY IMPRESSION IN FRONT OF HISTOLOGIC DIAGNOSIS IN THE BLADDER TUMORS. TO COINCIDE?”

OBJETIVE: How quality control in a university hospital and immediatily after to recents publications; we planed to evaluation of the correlation of cystoscopy impression with the histologic diagnosis after of transurethral resection (TUR).

MATERIAL AND METHODS: To give more truthfulness to the study, we requested to all departament member's, that to base in your experience to describe the endoscopic characteric of the next bladder tumors groups: superficial and low-grade GI-II Ta, superficial and high-grade GIII Ta and high grade and/or T1-<T2. In a total of 172 patients, we evaluated the initial cystoscopy impression and we to compared it with histologic diagnosis after to TUR.

RESULTS: In 172 tumors the cystoscopy clasifed in 69 cases how superficial and low grade GI-II Ta-T1, 40 how superficial and high grade and 55 how high grade and/or invasive tumors GIII T1-<T2. When, we compared it with the histologic diagnosis, the cystoscopy to coincided in 46 de 69 cases (66.6\%) (PNS) with the group of low-grade GI-II Ta$\mathrm{T} 1$ in 13 of 40 (32.5\%) (P<0.005) with the group of superficial high grade GIII Ta and 45 of 51 (88.2\%) (PNS) with the group of GIII T1 and/or invasive tumors. In 15 of 172 the endoscopic description its not conclusive. And finally in 12 cases the histologic diagnosis were normal.

CONCLUSIONS: In order of this results, we to consider that in a university hospital is essential the histologic diagnosis before any therapeutic decission, because the initial cystoscopy impression have a low correlation with the histologic diagnosis. We are disagreement with recent publication that propose the outpatient cystoscopy with fulguration to base only to the cystoscopy impression.

KEY WORDS: Bladder tumors. Endoscopic impression. Histologic diagnosis. 
$\mathrm{L}^{2}$ a medicina es un arte, afirmaban filosóficamente médicos que nos han precedido, ellos encontraban en la semiología un arma, quizás, la más importante para establecer un diagnóstico. La primera característica de un médico es que ha de ser observador y por todos es sabido que el primer paso semiológico es la inspección. Para el urólogo, así como para otras especialidades que en su práctica diaria utilizan aparatos endoscópicos, la inspección va más allá, pues podemos aplicarla tanto externa como internamente y es por ésto que actualmente han surgido términos como el de "Semiología Endoscópica".

Los tumores vesicales presentan características morfológicas que pueden sugerir al endoscopista la superficialidad o no y un poco menos el grado de agresividad de los mismos. Esto sin embargo, no significa que se pueda prescindir de una valoración histológica antes de una decisión terapéutica definitiva.

Como control de calidad en un hospital docente como el nuestro y a raíz de publicaciones recientes al respecto ${ }^{6}$. nos propusimos evaluar la correlación existente entre la impresión cistoscópica de los tumores vesicales y el diagnóstico histológico posterior a la resección transuretral (RTU).

\section{MATERIAL Y MÉTODOS}

Realizamos un estudio retrospectivo y descriptivo en 172 pacientes afectos de tumores vesicales, diagnosticados durante los años 2000 y 2001 para valorar la impresión cistoscópica inicial y compararlo con el diagnóstico histológico tras la RTU. Se clasificaron a los pacientes dependiendo del estadio y grado de tumor vesical en tres grupos:

1. Tumores superficiales de bajo grado (T.S.B.G.) que incluían GI-II, Ta-T1.

2. Tumores superficiales de alto grado (T.S.A.G.) que incluía GIII, Ta.

3. Tumores de alto grado y/o invasivos en donde incluimos GIII, T1-T2.

Intentando darle mayor objetividad al estudio, se solicitó a los miembros del servicio (tanto staff como residentes) que respondieran a la siguiente encuesta:

1. ¿Cree usted que la cistoscopia puede distinguir los tumores de bajo grado, de los de alto grado y los superficiales de los infiltrantes?

Respuesta: Si
2. En caso afirmativo, con respecto a la semiología endoscópica (papilas, tamaño, base multiplicidad, etc.) y basado en su experiencia, ¿como describiría los siguientes grupos de tumores?

a) ¿Tumores superficiales de bajo grado?

Respuesta: papila fina, base estrecha, pediculados, sin calcificaciones ni necrosis, sin alteraciones peritumorales, translúcidos, poca vascularización y exofíticos.

b) ¿Tumores superficiales de alto grado?

Respuesta: poco exofíticos, de base amplia, mal delimitados, aterciopelados, papilas gruesas poco ramificadas, con focos de necrosis, fibrina y calcificaciones, de morfología sólida papilar.

c) ¿Tumores de alto grado, estadios $T 1 \mathrm{y} / \mathrm{o}$ invasivos?

Respuesta: base ancha, áreas de necrosis, mucosa peritumoral edematosa, hiperémica, neovascularización importante, papila gruesa, aspecto sólido, poco móviles, poco exofíticos, con fibrina y calcificaciones.

Posteriormente, se revisaron las hojas operatorias de la cistoscopia del diagnóstico inicial y se clasificaron de acuerdo al cuestionario en los 3 grupos anteriormente mencionados. Seguidamente, se revisó el diagnóstico histológico tras la RTU y finalmente se compararon ambos, es decir, impresión cistoscópica inicial vr diagnóstico histológico tras la RTU. Hemos de mencionar que la revisión endoscópica no fue realizada por una sola persona, y en ello participaron los diversos miembros del staff y residentes (en el último caso siempre fueron supervisados por un miembro del staff).

\section{RESULTADOS}

De los 172 tumores (total de casos), la cistoscopia inicial clasificó a 69 pacientes en el grupo T.S.B.G., coincidiendo con el diagnóstico histológico tras la RTU en 46 casos $(66,6 \%$ ) (PNS) y no hubo correlación en los otros 23 casos, entre los cuales llama la atención 12 casos cuyo diagnóstico histológico descartó tumor (Fig. 1). De igual forma 40 pacientes fueron clasificados en el grupo T.S.A.G. coincidiendo únicamente en 13 casos $(32,5 \%)(\mathrm{P}<0,005)$. Este grupo es sin duda el de mayor dificultad para el diagnóstico endoscópico siendo clasificados $21 / 40$ como T.S.B.G., lo que corresponde a un 52\% de infraestadiaje, por otra parte en 1 caso el diagnóstico histológico descartó 


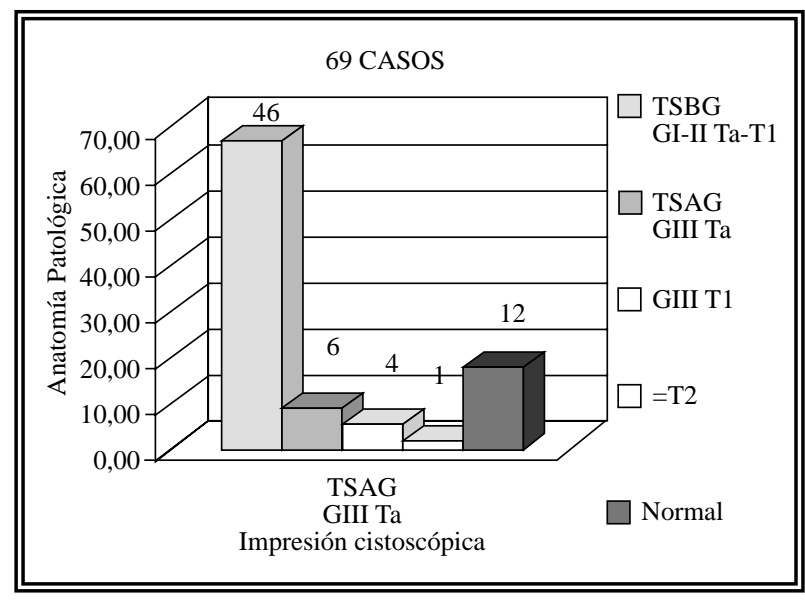

FIGURA 1. Correlación entre la impresión cistoscópica y el diagnóstico histológico final (46/69), en el grupo de pacientes con hallazgos endoscópicos compatibles con tumores superficiales de bajo grado (T.S.B.G.).

neoformación (Fig. 2). Finalmente 55 pacientes fueron clasificados en el grupo GIII T1 y/o invasivos coincidiendo la impresión cistoscópica con la histología final en 45 casos $(88,2 \%)$ (PNS). Nos llamó la atención la similitud de la morfología y características tumorales del grupo GIII T1 con respecto a los tumores infiltrantes, y es por ésto que decidimos en el resultado final agruparlos en uno solo; además, en este grupo en ningún caso el diagnóstico histológico descartó neoformación (Fig. 3). Del total de casos, en 15 los datos recogidos en la descripción endoscópica no fueron concluyentes para incluirlos en algún grupo. En 13 el

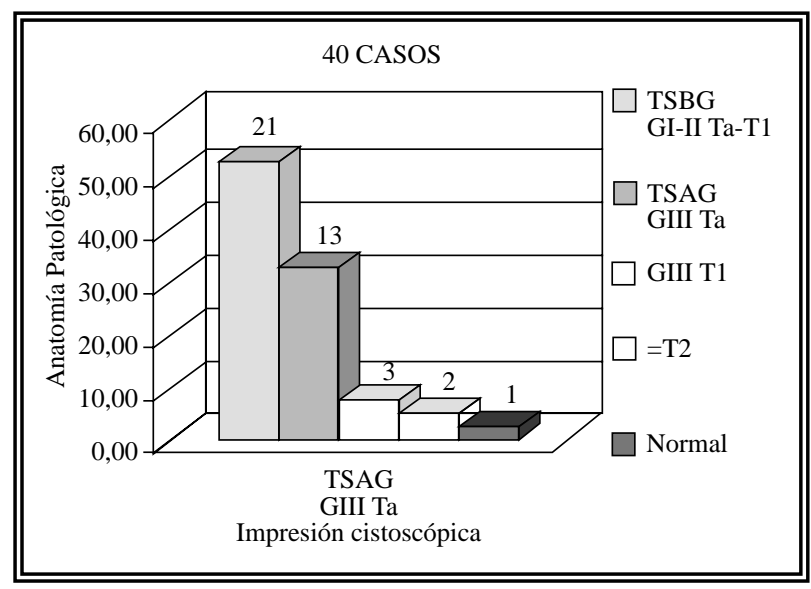

FIGURA 2. Correlación entre la impresión cistoscópica y el diagnóstico histológico final (13/40), en grupo de pacientes con hallazgos endoscópicos compatibles con tumores superficiales de alto grado (T.A.B.G.).

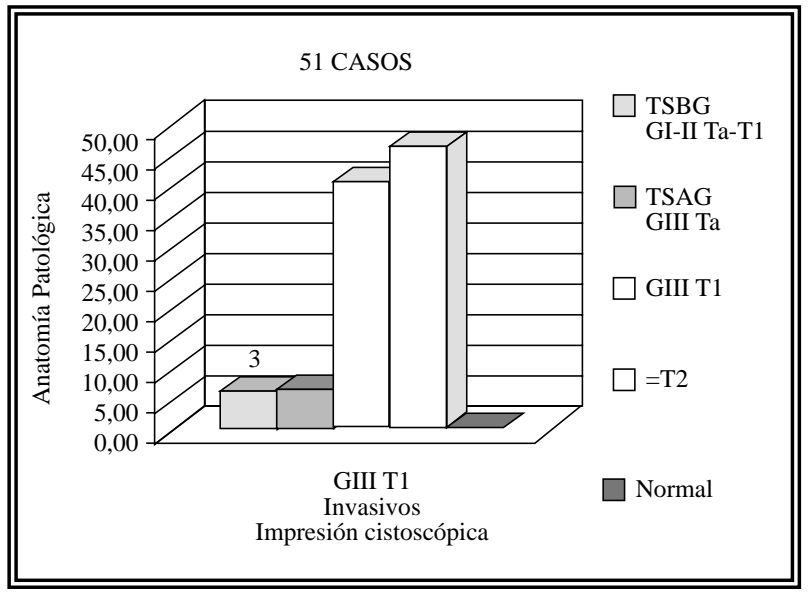

FIGURA 3. Correlación entre la impresión cistoscópica y el diagnóstico histológico final (45/51), en el grupo de pacientes con hallazgos endoscópicos compatibles con tumores de alto grado GIII T1 y/o infiltrantes.

diagnóstico histológico fue "ausencia de células tumorales" (Tabla I). Finalmente el resultado global fue $60,47 \%$ (104/172) de coincidencia, 30,8\% (51/172) no coincidieron y $8,72 \%$ los datos fueron insuficientes ( 15 casos) (Tabla II). El test comparativo empleado fue $\mathrm{x} 2$.

\section{DISCUSIÓN}

A pesar de que la cistoscopia es un procedimiento rutinario en el seguimiento de los tumores vesicales, hemos encontrado en la literatura médica pocas publicaciones que correlacionen el aspecto endoscópico con el diagnóstico histológico definitivo. Se ha descrito que la cistoscopia puede determinar la naturaleza maligna o no de una lesión vesical, sin embargo, el diagnóstico histológico de la biopsia es esencial para establecer el grado y estadio de la neoplasia ${ }^{1}$. Otros estudios han comparado el diagnóstico histológico tras la RTU con las piezas de la cistectomía ${ }^{2}$. Existen estudios que comparan la cistoscopia con técnicas de imagen combinadas como por ejemplo la ecografía abdominal y la transrectal con el fin de reducir el número de cistoscopias ${ }^{3,4}$. Un estudio reciente propone la fulguración endoscópica ambulatoriamente en el seguimiento de las recidivas de los tumores vesicales de bajo grado y estadio, no considerando esencial la biopsia y por consiguiente el diagnóstico histológico en este grupo de tumores $^{5,6}$. Basados en nuestros resultados consideramos que en un hospital docente como el nuestro 
TABLA I

\begin{tabular}{|l|c|c|c|c|c|c|}
\hline \hline \multicolumn{2}{|l|}{ VALORACIÓN CISTOSCÓPICA } & \multicolumn{3}{|c|}{ ANATOMÍA PATOLÓGICA } \\
\hline $\begin{array}{l}\text { Grupos } \\
\text { tumorales }\end{array}$ & $\begin{array}{c}\text { No } \\
\text { Ptes. }\end{array}$ & $\begin{array}{c}\text { T.S.B.G. } \\
\text { GI-II Ta }\end{array}$ & $\begin{array}{c}\text { T.S.A.G. } \\
\text { GIII Ta }\end{array}$ & GIII T1 & $\geq$ T2 & $\begin{array}{c}\text { A.P. } \\
\text { NL }\end{array}$ \\
\hline $\begin{array}{l}\text { T.S.B.G. } \\
\text { GI-II Ta-T1 }\end{array}$ & 69 & $\mathbf{4 6}$ & 6 & 4 & 12 \\
\hline $\begin{array}{l}\text { T.S.A.G. } \\
\text { GIII Ta }\end{array}$ & 40 & 21 & $\mathbf{1 3}$ & 3 & 2 & 1 \\
\hline $\begin{array}{l}\text { GIII T1 } \\
\text { Invasivos }\end{array}$ & 51 & 3 & 3 & $\mathbf{2 1}$ & $\mathbf{2 4}$ & 0 \\
\hline
\end{tabular}

Presenta de forma resumida la correlación cistoscópica a la izquierda vs diagnóstico histológico a la derecha. En negritas el número de pacientes que coincidieron en los diferentes grupos tumorales.

\section{TABLA II}

\begin{tabular}{|l|c|c|}
\hline $\begin{array}{l}\text { Correlación } \\
\text { cistoscópica vs A.P. }\end{array}$ & No casos & Porcentajes \\
\hline Correlación & 104 & $60,47 \%$ \\
\hline No correlación & 53 & $30,81 \%$ \\
\hline $\begin{array}{l}\text { Cistoscopia con } \\
\text { descripción insuficiente }\end{array}$ & 15 & $8,72 \%$ \\
\hline Total & 172 & $100 \%$ \\
\hline
\end{tabular}

Resultado general de correlación cistoscópica vs diagnóstico histológico.

la correlación entre la impresión cistoscópica y el diagnóstico histológico es baja, y por lo tanto el diagnóstico histológico es imprescindible antes de tomar una decisión terapéutica definitiva, tanto en el paciente diagnosticado por primera vez como en el seguimiento de los tumores vesicales, aún en los de bajo grado y estadio. Sin embargo, hay que tener en cuenta que la cistoscopia fue realizada por varios urólogos entre ellos residentes (aunque éstos siempre fueron supervisados por un miembro del staff), a diferencia del estudio antes descrito en el que la cistoscopia fue realizada por una sola persona. De todas maneras estamos en desacuerdo en realizar una fulguración endoscópica de las recidivas tumorales de bajo grado y estadio basándose solamente en la impresión cistoscópica, pues es sabido que éstos pueden progresar de grado y con esta técnica no se dispone de material para estudio histológico. Dejamos la inquietud para la autoevaluación y que el arte se siga cultivando.

\section{REFERENCIAS}

1. ENDRIZZI J, CINA S, HARMON W et al.: Correlation of cistoscopic impression and histologic diagnosis on biopsy specimens of the bladder. J Urol 2000; 163: 134 (A588).

2. CHENG L, NEUMANN RM, WEAVER AL et al.: Grading and staging bladder carcinoma in transurethral resection specimens: correlation with 105 matched cystectomy specimens. Am J Clin Pathol 2000; 113: 275-279.

3. DAVIES AH, MASTORAKOU I, DICKSON AJ, CRANSTON D et al.: Detection of recurrent bladder tumours by transrectal and abdominal ultrasound compared with cystoscopy. Br J Urol 1989; 64: 409-411.

4. DAVIES AH, CRANSTON D, MEAGHER T et al.: Flexible cystoscopy compared with ultrasound in the detection of recurrent bladder tumours. $\mathrm{Br} \mathrm{J} \mathrm{Urol}$ 1991; 67: 491-492.

5. HERR HW.: Outpatient flexible cystoscopy with fulguration in the management of recurrent bladder tumors. J Urol 1990; 144: 1.365-1.366.

6. HERR HW.: Does cystectomy correlate with the histology of recurrent papillary tumours of the bladder?. Br J Urol 2001; 88: 683-685.

Dr. E. de León Morales

Servicio de Urología. Hospital del Mar

Paseo Marítimo, 25-29

08003 Barcelona

(Trabajo recibido el 3 julio de 2002) 